\title{
Lung Volume Determination by Dual-source Computed Tomography in Infants with Pulmonary Artery Sling
}

\author{
Hao Yang ${ }^{1}$, Wen Bing ${ }^{2}$, Qiuyi $\mathrm{Cai}^{1}$, Jianlin $\mathrm{Li}^{1}$, and Liangbo $\mathrm{Hu}^{3}$ \\ ${ }^{1}$ Chengdu Third People's Hospital \\ ${ }^{2}$ Yiyang Central Hospital \\ ${ }^{3}$ Yongchuan Hospital of Chongqing Medical University
}

April 7, 2021

\begin{abstract}
Background: Pulmonary artery sling (PAS) is associated with tracheal stenosis and left pulmonary artery (LPA) dysplasia in infants, which may cause pulmonary dysplasia and lung volume variations. We aimed to assess these altered lung volumes in patients with PAS using dual-source computed tomography (DSCT). Methods: We retrospectively enrolled patients with surgically confirmed PAS and compared them with matched normal controls. All participants underwent DSCT examination. We measured and compared the diameters of the trachea, main bronchus and main pulmonary artery (MPA) and its branches and both lung volumes on axial and reconstructed computed tomography images. Results: There were no statistical differences in the diameters of MPA or right pulmonary artery between patients and controls. The diameters of the stenosed trachea , left and right main bronchi and LPA were smaller in the PAS group than in the control group ( $<<0.001)$, and significant differences were evident in the left lung volume $(\mathrm{p}<0.001)$, right lung volume $(\mathrm{p}=0.008)$ and the right-to-left lung volume ratio $(\mathrm{p}=0.004)$ between the two groups. Pearson's correlation and linear regression analyses between the diameters of the trachea and MPA, total lung volume, ipsilateral bronchial and pulmonary artery branches and ipsilateral lung volume ranged from 0.71 to $0.87(\mathrm{p}<0.001)$ and 0.57 to $0.77(\mathrm{p}<0.05)$ for the control and PAS groups, respectively. Conclusions: Lung development is disordered in patients with PAS, which causes not only tracheal stenosis and LPA dysplasia but also dysplasia of both lungs.
\end{abstract}

\section{Hosted file}

Manuscript.pdf available at https://authorea.com/users/406200/articles/516949-lung-volumedetermination-by-dual-source-computed-tomography-in-infants-with-pulmonary-artery-sling 

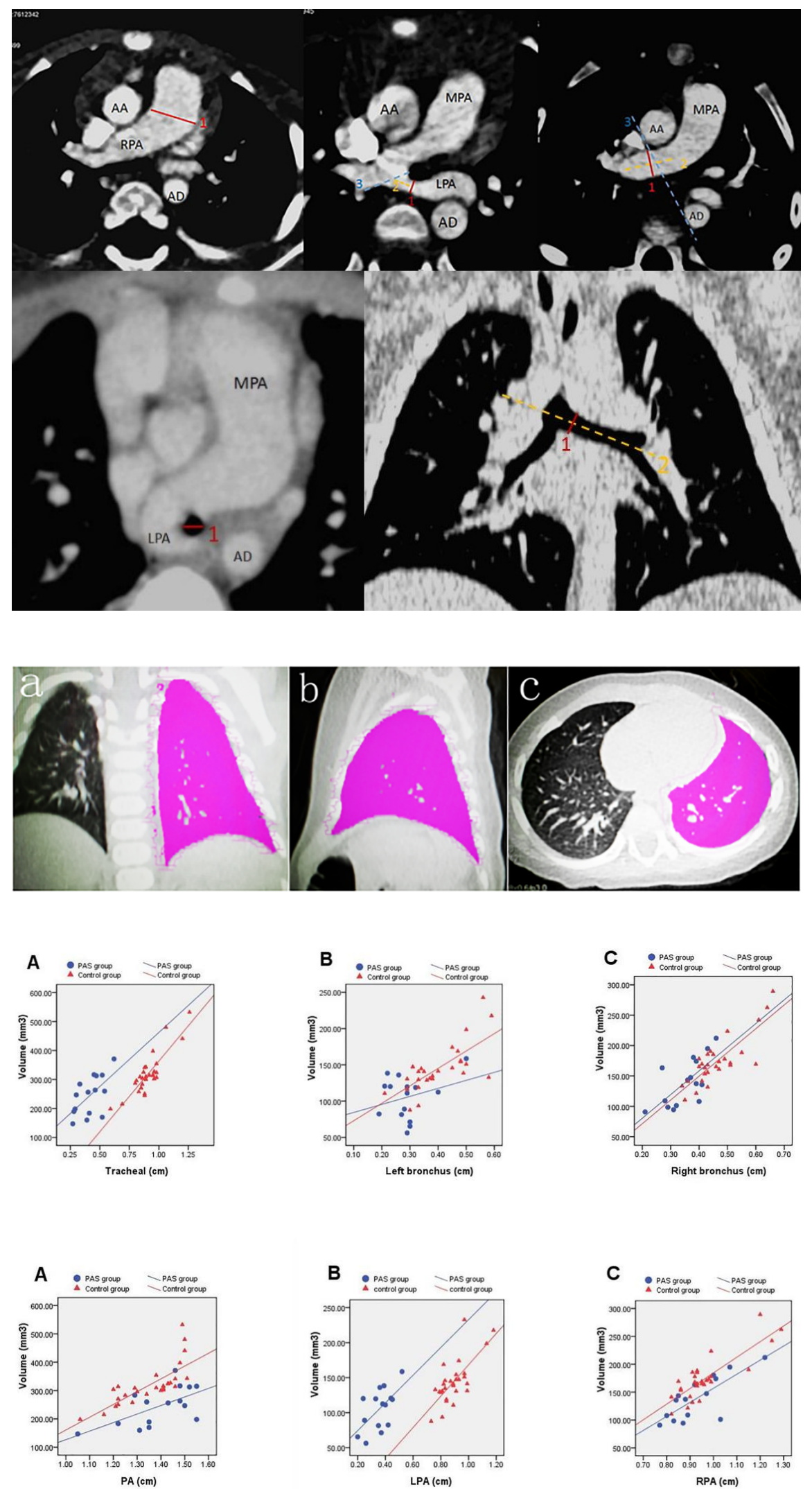